\title{
INVESTIGACIONES
}

\section{Evaluación de programas educativos sobre Patrimonio Cultural Inmaterial ${ }^{*}$}

\author{
Evaluation of educational programmes on Intangible Cultural Heritage
}

\author{
Olaia Fontal, ${ }^{a}$ Marta Martínez $z^{a b}$ \\ aDepartamento de didáctica de la expresión musical, plástica y corporal, Universidad de Valladolid \\ Telf.: (34) 983084063. Correo electrónico: olaia.fontal@uva.es \\ 'Telf.: (34) 983423745. Correo electrónico: marta.martinez.rodriguez@uva.es
}

\begin{abstract}
El artículo ofrece un análisis relacional de los enfoques y categorías de programas educativos generados en torno al patrimonio inmaterial, derivado de un estudio financiado por el Ministerio de Economía y Competitividad y los Fondos Feder (UE) en el marco del proyecto de investigación EDU2015-65716-C2-2-R, y se enmarca en las acciones del Plan Nacional de Educación y Patrimonio. Este análisis se desarrolla dentro de un proceso evaluativo con una muestra de 202 programas educativos, que tiene como finalidad detectar el estado de la cuestión, así como sus fortalezas y debilidades. El estudio muestra cómo los programas analizados abordan todos los ámbitos educativos, y presentan una amplia gama de tipologías. También se observan tendencias relacionales entre el tipo de público y tipo de programa. Por otro lado, se denotan deficiencias en torno al alto porcentaje de programas detectados sin diseño educativo. Este aspecto nos plantea la necesidad de generar nuevas prospectivas para la educación patrimonial, que garanticen su función activadora de los procesos de identización y de la sociedad hacia su patrimonio.
\end{abstract}

Palabras clave: educación patrimonial, patrimonio cultural inmaterial, evaluación de programa, UNESCO, Instituto del patrimonio cultural de España.

\begin{abstract}
The paper provides a relational analysis of approaches and categories in educational programs related to intangible heritage, in the framework of a study funded by Economy and Competitivity Ministry in Spain and Feder funds (UE) based on the Research EDU2015-65716-C2-2-R and is part of a set of actions promoted by the National Plan for Heritage and Education. Our analysis is conducted within an evaluation process applied on a sample of 202 educational programmes in order to diagnose the state of the question and detect their strengths and weaknesses. The study shows that the educational programmes under scrutiny deal with all educational settings and belong to a broad range of typologies. Additionally, relational tendencies are established between the programmes' target audience and their typology. On the other hand, shortfalls are detected involving a high percentage of programmes that have no educational design. On the whole, there emerges the need to generate new perspectives for heritage education that ensure its activating function of the processes of identity and society towards its heritage.
\end{abstract}

Keywords: heritage education, intangible cultural heritage, programme evaluation, UNESCO, Spain's Institute for Cultural Heritage.

Este trabajo ha sido financiado por el Ministerio de Economía y Competitividad y con Fondos Feder (UE) en el marco del proyecto de investigación EDU2015-65716-C2-2-R. 


\section{INTRODUCCIÓN}

El patrimonio inmaterial, aunque ya entendido como un concepto asentado y reconocido institucionalmente a nivel internacional por diversos organismos como la UNESCO o el Consejo de Europa, puede ser aún considerado un patrimonio emergente, si tenemos en cuenta el dinamismo intrínseco inserto en la conceptualización del término patrimonio cultural. Nos estamos refiriendo a la múltiple y variable dimensión de valores atribuidos por las diferentes sociedades hacia los bienes culturales a lo largo de la historia (Teixeira, 2006). Entendemos así que esta conceptualización seguirá avanzando en un sentido rizomático, crecerá y evolucionará indefinidamente, generando nuevos elementos y valores, los cuales no seguirán una subordinación jerárquica, sino que "cualquier elemento podrá incidir en cualquier otro" (Deleuze \& Guattari, 1972, p. 13). Si echamos la vista atrás, podemos observar, desde una posición privilegiada, el camino recorrido por el concepto del patrimonio cultural, que confirma de algún modo la continua evolución del término. Este camino está marcado por la incorporación del concepto antropológico de cultura a la gestión del patrimonio, que fluye en paralelo al auge de los movimientos sociales en pro del reconocimiento de su especificidad cultural (Carrera, 2005). Estos dos aspectos son determinantes en el origen de nuevos valores identitarios atribuibles a otro tipo de bienes culturales centrados en las expresiones y manifestaciones de una comunidad, originadas como resultado de la adaptación e interacción con el entorno. Así surge la incorporación de la dimensión intangible, que permite conocer la significación social de los bienes, "promover los vínculos entre ellos y dentro de ellos y comprender la vigencia y transformaciones de este 'todo' vivo que es el Patrimonio Cultural" (González, 2015, p. 6). Por ello, la definición de patrimonio cultural es "siempre abierta, negociable, dialógica, transformadora y reflexiva" (Santamarina, 2005, p. 48), en la que participan distintos operadores sociales.

Nos preguntamos entonces si la razón nos permite establecer alguna norma fija o tipo de regla, quizás a través de la comparación de tendencias o la relación de ideas, que nos permita vislumbrar el camino futuro sobre aquello que será o deberá ser considerado patrimonio. $\mathrm{Si}$ apelamos a un pensamiento filosófico, podremos proponer basar el fundamento en las ciencias prácticas, en la experiencia (Hume, 1757). Huimos entonces de atribuir los valores a una condición intrínseca y objetiva del bien cultural, y ubicamos los valores fuera de este, otorgados por el hombre. Son estos valores atribuidos, derivados de la experiencia y las relaciones, los que finalmente convierten a los bienes culturales en patrimonio. En la medida en la cual asumimos que este es el único encargado de otorgar valores a dichos bienes, entendemos la multiplicidad de dimensiones y la posibilidad de miradas hacia el patrimonio, donde la educación es una mirada fundamental, ya que hablar de relaciones, experiencias y procesos es hablar de educación. Por lo tanto, "comprender el patrimonio en estos términos permite situar la acción educativa como algo no solo necesario sino absolutamente imprescindible, desde el momento en que la educación patrimonial opera con este tipo de procesos" (Fontal \& Valles, 2013, p. 151).

\section{ANTECEDENTES Y PROBLEMA DE ESTUDIO: EL BINOMIO PATRIMONIO Y EDUCACIÓN}

El volumen científico desarrollado en torno a la educación patrimonial ha aumentado de manera exponencial en la última década y continua en crecimiento si aludimos a la 
proliferación de investigaciones realizadas en este campo. Muchas de estas investigaciones se desarrollan dentro del ámbito formal de la educación y abordan diferentes perspectivas, como el análisis de la enseñanza del patrimonio en las ciencias sociales y el uso del patrimonio en los libros de texto (Cuenca \& López, 2014), o las concepciones de los profesores sobre patrimonio y educación patrimonial (Estepa, Ávila \& Ferreras, 2008; Jiménez, Cuenca \& Ferreras, 2010). También encontramos el desarrollo de manuales para ayudar a los profesores a integrar la educación patrimonial en las clases (De Troyer et al., 2005). Esta última investigación tuvo lugar dentro del Proyecto HEREDUC, desarrollado de manera cooperativa por Alemania, Bélgica, Francia, Holanda e Italia. Incluso, hallamos investigaciones que presentan la interpretación de los resultados de los programas sobre educación patrimonial implementados en la República Checa, bajo el título de programa NAKI (National and Cultural Identity) (Dušan, 2015). Dentro del ámbito formal también encontramos estudios que realizan un análisis de la normativa educativa con la intención de conocer el uso, el tratamiento y el enfoque que recibe en ellas el patrimonio (Cuenca, Estepa \& Martín, 2011; Fontal \& Martínez, 2016; Pinto \& Molina, 2015). Sus resultados muestran que el patrimonio es un contenido presente desarrollado de forma trasversal y siempre desde un sentido simbólico-identitario. Otras investigaciones proponen un análisis relacional entre el currículo de educación primaria y los planes de estudios de los futuros docentes (Fontal et al., 2017). También encontramos investigaciones que determinan hasta qué punto las diferentes áreas del currículo conciencian y fomentan el respeto hacia el patrimonio (Barghi et al., 2016) e investigaciones que analizan el grado de conciencia patrimonial y compromiso de los alumnos (Azmana et al., 2010; Curtis \& Seymour, 2004). Más estrechamente relacionadas con el patrimonio cultural inmaterial, encontramos estudios centrados en la educación universitaria (Jin-long, 2009), el punto de vista de los estudiantes de educación primaria y social (Yalcinkaya, 2015), y la adquisición y desarrollo del lenguaje (Asiáin \& Aznárez, 2012).

Las investigaciones desarrolladas en el ámbito informal se encuentran, en su mayor parte, vinculadas al entorno museal (Garner, Kaplan \& Pugh, 2016; Nardi, 2008; Rosmary \& Sutter, 2012) y abordan la educación como mediación cultural en los museos. Con un enfoque más relacional entre museo, comunidad, aprendizaje continuo e identidad, encontramos las investigaciones de Falk, Dierking y Adams (2011), y utilizando el patrimonio inmaterial como recurso emergente en la museología educativa a Yanes (2007). Entre la relación sobre museos, educación patrimonial y diversidad de colectivos hacemos referencia a Fontal y Marín (2016), que entienden la educación patrimonial desde una perspectiva inclusiva y realizan una evaluación de programas educativos adaptados a diversos públicos con la finalidad de desarrollar un modelo de inclusión universal. La utilización de recursos tecnológicos está cada vez más presente en los museos, en la educación y en la sociedad en general, provocando un aumento de investigaciones sobre educación patrimonial y nuevas tecnologías. Estas investigaciones abordan la educación patrimonial desde el m-learning (Angelopoulou et al., 2012; Ibáñez et al., 2012), el e-learning (Lobovikov-Katz et al., 2014), la aplicación didáctica de modelos digitales (Forte, 2007; Vicent, Rivero \& Feliu, 2015), y el concepto de edutainment y serious games (Haddad, 2016). Relacionadas de manera más específica con el patrimonio inmaterial, destacan las investigaciones realizadas dentro del proyecto i-Treasures, que se integra en el $7^{\circ}$ Programa Marco de la Unión Europea. Este proyecto ha generado una plataforma que contiene recursos educativos innovadores sobre patrimonio cultural inmaterial (Ott 
\& Pozzi, 2011; Ott, Dagnino \& Pozzi, 2015). Esta relación entre las TIC y el Patrimonio Cultural Inmaterial es la base del proyecto ICHPEDIA desarrollado en Corea, cuyo principal objetivo es elaborar un inventario nacional que sea utilizado para la difusión y sensibilización de esta tipología patrimonial (Soon, 2014). Finalmente, hacemos alusión a un proyecto desarrollado en Turquía dentro del ámbito formal, que plantea un programa educativo sobre patrimonio intangible utilizando la aplicación tecnológica educativa "WebQuest" (Kasapoglu, 2016).

Las investigaciones referidas están sustentadas sobre bases teóricas que han desarrollado un marco de referencia para una disciplina relativamente reciente, y que hasta hace varias décadas no disponía de bagaje teórico. A este respecto destacamos el estudio realizado por Fontal e Ibáñez (2017) que ofrece un análisis de la evolución de la investigación en educación patrimonial a través de indicadores de alto impacto y publicaciones de artículos en las bases de datos de referencia internacionales WOS y Scopus. Dentro de este marco de referencia destacan autores como Cuenca (2003), quien aborda la educación patrimonial desde la didáctica de las ciencias sociales y Fontal (2003) desde una perspectiva más identitaria. Copeland (2006) y Texeira (2006) plantean la educación patrimonial desde el gran potencial didáctico que presenta para trabajar la ciudadanía, la identidad y la democracia. Lobovikov-Katz (2009) entiende la educación patrimonial como una disciplina capaz de generar y garantizar procesos como la sensibilización o socialización del patrimonio cultural. En torno a la educación patrimonial y su didáctica, destacamos a Calaf (2009), por sentar las bases epistemológicas y Fontal (2008) que entiende la dimensión humana como un elemento imprescindible en la didáctica del patrimonio. Toda la literatura y las investigaciones desarrolladas están acrisoladas por el objetivo de situar la educación patrimonial como una disciplina esencial en la salvaguarda y gestión del patrimonio cultural, tanto tangible como intangible. Esto se debe a que entendemos la educación patrimonial como el motor que acciona la cadena de sensibilización, "conocer para comprender, comprender para respetar y respetar para valorar" (Fontal, 2003, p. 209). La educación patrimonial garantiza la atribución de valores a los bienes culturales por parte de la sociedad, pasando estos a formar parte de su patrimonio, que querrá cuidar, disfrutar y trasmitir. Muchos estudios defienden la posición de la educación patrimonial como disciplina fundamental para la gestión y conservación del patrimonio cultural (Fontal \& Juanola, 2015) e incluso para el beneficio social de la población (Buckley \& Graves, 2016), ya que entienden que la misma es capaz de garantizar procesos necesarios para trasformar los bienes culturales en patrimonio para la sociedad.

Este tema preocupa dentro de nuestro ámbito nacional a múltiples marcos normativos e institucionales. En el año 2006, España ratifica la Convención para la Salvaguarda del Patrimonio Inmaterial, que establece cinco ámbitos o contextos en los que se manifiesta: "las tradiciones y expresiones orales; las artes del espectáculo; los usos sociales; rituales y actos festivos; los conocimientos y usos relacionados con la naturaleza y el universo; y las técnicas artesanales tradicionales" (UNESCO, 2003, p. 2). Desde entonces se comienza a trabajar con la finalidad de cumplir los objetivos planteados en la convención. Este proceso culmina en 2011, con la entrada en vigor del Plan Nacional para la Salvaguarda del Patrimonio Cultural Inmaterial. Pero la preocupación en torno a esta materia continúa, y se desarrolla la Ley 10/2015, para la Salvaguardia del Patrimonio Cultural Inmaterial. Ambas normativas nacionales perfilan los ámbitos o contextos en los que se manifiesta el Patrimonio Cultural Inmaterial (González \& Querol, 2014). 
Dentro de estas normativas se presentan medidas educativas que instan a las administraciones educativas a velar por la inclusión del conocimiento y respeto al patrimonio inmaterial en los contenidos educativos. Estas nuevas medidas nos recuerdan la importancia de la educación para la gestión y conservación del patrimonio, ya planteada en 2013 en el Plan Nacional de Educación y Patrimonio (en adelante PNEyP) (Domingo, Fontal \& Ballesteros, 2013). Dentro de las acciones del PNEyP, financiado por el Instituto del Patrimonio Cultural de España (IPCE), se desarrolla el presente estudio requerido para la consecución de sus objetivos en el marco del proyecto de investigación EDU2015$65716-\mathrm{C} 2-2 \mathrm{R}^{1}$.

Este estudio está estrechamente vinculado con los objetivos del PNEyP, ya que busca cumplir y garantizar la investigación continua y el seguimiento de las acciones y líneas proyectuales desarrolladas en materia de educación patrimonial (Domingo et al., 2013). La investigación responde a una evaluación secuenciada de una selección de programas educativos sobre patrimonio cultural intangible, que surge ante la necesidad de investigación en torno al mismo debido al reconocimiento legislativo más reciente en comparación con otras tipologías patrimoniales, y cuyas acciones educativas necesitan -muchas de ellas de gran valor pedagógico- ser categorizadas y analizadas. La finalidad del mismo es conocer el estado de la cuestión, así como las fortalezas y debilidades de los programas educativos, que ayuden a establecer criterios y parámetros para el diseño de materiales educativos estructurados que permitan adaptarse a múltiples contextos y colectivos, superando el modelo lineal y cerrado. Esta evaluación secuenciada de programas consta de varias fases. En el presente artículo se presentan los resultados de la primera fase de evaluación relativa al análisis estadístico-descriptivo y correlacional de los programas, que pretende conocer sus enfoques y categorías. Este aspecto nos permitirá vislumbrar el estado de la cuestión en un campo escasamente analizado, y ayudará a definir los estándares de calidad relacionados con el diseño, la implementación y los resultados (Stake, 2006).

\section{EL MÉTODO OEPE}

El estudio que presentamos forma parte de una investigación más amplia dentro del Observatorio de Educación Patrimonial en España. El observatorio ha definido un método (Fontal, 2016) para inventariar, analizar y evaluar programas educativos, y tiene entre sus objetivos dar a conocer sus resultados, que ayudan a diagnosticar el estado de la educación patrimonial, tanto en España como en el ámbito internacional (Fontal \& Gómez-Redondo, 2016; Fontal \& Marín, 2016; Marín et al., 2017). Desde el Observatorio, en el momento actual, hay inventariados en la base de datos digital para investigadores $\mathrm{n}=1682$ programas de educación patrimonial desarrollados en el ámbito de la educación formal, no formal e informal, tanto nacionales como internacionales.

Título: "Evaluación de los aprendizajes en programas de educación patrimonial centrados en los procesos de sensibilización, valorización y socialización del patrimonio cultural". Fecha de concesión: 1-1-2016 a 31-12-2018. IP: Olaia Fontal Merillas. Dentro del Programa Estatal de Investigación, Desarrollo e Innovación Orientada a los Retos de la Sociedad, Convocatoria 2015, Modalidad 1: «Proyectos de I+D+I». Entidades financiadoras: MINECO + FONDOS FEDER. 


\subsection{CRITERIOS PARA LA SELECCIÓN DE LA MUESTRA}

La muestra de nuestra investigación se corresponde con un muestreo intencional que ha sido entresacado de la base de datos digital del OEPE, realizando una selección de nuestros descriptores de interés: pertenencia a la categoría de Patrimonio Inmaterial y país de localización: España. Estos programas han sido localizados empleando términos y descriptores del campo semántico relativos al PCI, y utilizando buscadores oficiales del Ministerio de Educación Cultura y Deporte, así como buscadores genéricos. Los programas resultantes son sometidos a una serie de criterios de inclusión y exclusión (Tabla I).

Tabla 1. Criterios de inclusión y exclusión de los programas educativos para el inventario

\begin{tabular}{|c|c|}
\hline Criterios de Inclusión & Criterios de Exclusión \\
\hline $\begin{array}{l}\text { Contener el término patrimonio cultural } \\
\text { inmaterial o los ámbitos del PCI que establece la } \\
\text { Convención UNESCO } 2003 \text {. }\end{array}$ & Web o portal que únicamente informa. \\
\hline Contener algún término afín al ámbito educativo. & Se trata únicamente de material divulgativo. \\
\hline $\begin{array}{l}\text { Actividad que realiza un museo dentro del ámbito } \\
\text { formal o el ámbito informal. }\end{array}$ & $\begin{array}{l}\text { Webs de museos. Si sus propuestas no son } \\
\text { referidas al patrimonio. }\end{array}$ \\
\hline $\begin{array}{l}\text { Organizado por una institución pública, privada o } \\
\text { que implique varias entidades. }\end{array}$ & \\
\hline $\begin{array}{l}\text { Generado en España, ámbito nacional, autonómico, } \\
\text { regional o local. }\end{array}$ & \\
\hline Diseño educativo con objetivos. & \\
\hline Se dirige a más de un público. & \\
\hline $\begin{array}{l}\text { Se dirige, de forma explícita, a públicos con } \\
\text { necesidades educativas específicas. }\end{array}$ & \\
\hline Se trata de un curso o programa de formación. & \\
\hline $\begin{array}{l}\text { Formato web con interacción de aprendizaje o } \\
\text { propuestas al usuario. }\end{array}$ & \\
\hline
\end{tabular}

Fuente: elaboración propia.

Así, de los 223 programas localizados, y después de someter los mismos a los criterios de inclusión y exclusión, obtenemos una muestra de 202 programas que procedemos a inventariar y que constituyen la muestra final a analizar.

\subsection{INSTRUMENTOS DE INVENTARIO Y ANÁLISIS}

El método cuenta con una ficha de inventario basada en descriptores para facilitar el registro de los programas y su gestión. Este instrumento de registro esta refrendado por su contenido, pues dispone de todos aquellos campos necesarios para plasmar todas las categorías propias 
de un programa educativo. Los datos recogidos en las fichas de inventario son exportamos a una hoja Excel, y posteriormente al programa de análisis estadístico SPSS (Versión 21.0), que nos permite obtener las frecuencias buscadas, así como valores porcentuales.

\subsubsection{La ficha de inventario}

La ficha para el inventario de programas está organizada y sistematizada en 42 campos, y abarca los elementos más significativos del diseño educativo de cada programa. La organización consta de cuatro grandes apartados:

- Localización e identificación: En este primer apartado se clasifica e identifica el proyecto, se le asigna un nombre o título específico que posteriormente permita su búsqueda a través del programa diseñado; lugar donde se realiza el programa, ciudad y comunidad; datos de los responsables del proyecto y a qué institución u organización pertenecen. Se describen los datos de contacto con los organizadores y responsables, así como la página web en el caso de tenerla.

- Descripción del programa: En la descripción del programa se desarrollan los aspectos básicos: tipología de patrimonio, tipología de proyecto, adaptación a discapacitados y tipo, breve descripción, duración, lugar, profesionales encargados, disposición de documentos físicos y repercusiones sociales y mediáticas de la programación.

- Diseño educativo: En este apartado se describen los diferentes contenidos sobre la estructura didáctica del proyecto. Se introducen los datos relativos a las características educativas del programa: justificación del proyecto, objetivos, contenidos principales, orientación metodológica, estrategias de enseñanza aprendizaje, recursos empleados, datos sobre el educador que implementa el programa, sistemas y las herramientas de evaluación, así como la temporalización del mismo y la repercusión social y mediática que ha podido tener con posterioridad.

- Dosier documental: Recopilación de todos los documentos que aporta el programa, memoria, imágenes, videos, materiales didácticos, materiales de evaluación y cualquier otro documento disponible.

\subsection{PROCEDIMIENTO}

Este proceso secuencial, desde la búsqueda hasta la evaluación, se divide en cuatro partes diferenciadas:

- Búsqueda y localización de programas conforme a una serie de indicadores de búsqueda (Tabla 1).

- Discriminación. De la relación de 223 programas localizados, tras la aplicación de los criterios de inclusión y de exclusión, se obtiene una muestra 202 programas.

- Inventario. Se utiliza la ficha digital de recogida de datos, completando todos los campos y descriptores. Los programas son inventariados en base a su tipología de programa, en función de la mayor consistencia y conceptualización de su diseño educativo (Tabla 2), y se clasifican en base a 16 tipologías en función de su naturaleza educativa: programa educativo, proyecto educativo, diseño educativo, 
plan educativo, acción educativa, proyecto de mejora, actividad aislada, taller, recurso didáctico, herramienta didáctica, ruta o itinerario didáctico, curso, jornada, congreso, seminario y concurso.

- Análisis. Los datos inventariados son exportados al programa de análisis estadístico, donde realizamos, en primer lugar, un análisis estadístico-descriptivo y, posteriormente, un análisis correlacional.

Análisis estadístico-descriptivo. Se analizan los programas atendiendo a:
a. Tipo de programa en función de las 16 acciones educativas.
b. Ámbito donde se desarrolla, educación formal, no formal e informal.
c. Tipo de público al que se dirige, alumnado o público en general.
d. Adaptación a personas con diversidad funcional.
e. Diseño educativo.

Análisis correlacional. Se analiza la relación entre las siguientes variables:
a. Tipo de proyecto * Tipo de público.
b. Ámbito del programa * diseño educativo.

\section{RESULTADOS}

Los programas educativos como conjunto de procesos, proyectos, intervenciones, planificaciones, acciones, actividades y estudios de carácter didáctico, dirigidos hacia la consecución de unos objetivos previamente fijados, constituyen la muestra de los 202 programas sobre PCI. Son la base a través de la cual vamos a analizar sus enfoques y categorías.

\subsection{TIPOLOGÍA DE PROGRAMA EDUCATIVO}

Tras declarar el PNSPCI (2011) como valores a proteger las formas de vida, las prácticas sociales, los conocimientos, las técnicas y las mentalidades de los diversos individuos y grupos, la generalización de los términos "intangible" o "inmaterial" durante los últimos años da sus frutos a la vista del reconocimiento de la doble realidad, o como las corrientes teóricas apuntan, ampliando los horizontes de la conceptualización del término patrimonio cultural (Fontal, 2016). Este desarrollo se manifiesta también en el ámbito de la educación patrimonial, con la aparición en la última década de múltiples y diversos programas educativos sobre patrimonio cultural inmaterial, existiendo representación en todas las tipologías de programas. Si los objetivos de los distintos programas se establecen en función de su tipología -ya que estas presentan diferentes niveles de conceptualización-, cabe preguntarse cuáles son las tipologías más utilizadas actualmente (Tabla 2). 
Tabla 2. Frecuencias y porcentajes de los tipos de los programas educativos

\begin{tabular}{|c|c|c|c|c|c|}
\hline \multicolumn{2}{|r|}{ Tipo de Proyecto } & & & Porcentaje & Porcentaje \\
\hline \multirow{17}{*}{ Válidos } & Programa educativo & 41 & 20,3 & 20,3 & 20,3 \\
\hline & Proyecto educativo & 27 & 13,4 & 13,4 & 33,7 \\
\hline & Diseño didáctico & 30 & 14,9 & 14,9 & 48,5 \\
\hline & Recurso didáctico & 15 & 7,4 & 7,4 & 55,9 \\
\hline & Herramientas didácticas & 3 & 1,5 & 1,5 & 57,4 \\
\hline & Acción educativa & 17 & 8,4 & 8,4 & 65,8 \\
\hline & Actividad aislada & 7 & 3,5 & 3,5 & 69,3 \\
\hline & Taller & 20 & 9,9 & 9,9 & 79,2 \\
\hline & Proyecto de mejora & 4 & 2,0 & 2,0 & 81,2 \\
\hline & Concurso & 10 & 5,0 & 5,0 & 86,1 \\
\hline & Itinerario didáctico & 16 & 7,9 & 7,9 & 94,1 \\
\hline & Plan educativo & 2 & 1,0 & 1,0 & 95,0 \\
\hline & Congreso & 1 &, 5 &, 5 & 95,5 \\
\hline & Jornadas & 3 & 1,5 & 1,5 & 97,0 \\
\hline & Seminario & 2 & 1,0 & 1,0 & 98,0 \\
\hline & Curso & 4 & 2,0 & 2,0 & 100,0 \\
\hline & Total & 202 & 100,0 & 100,0 & \\
\hline
\end{tabular}

Fuente: elaboración propia.

El porcentaje más alto $(20,3 \%)$ corresponde a los programas educativos, seguido por los diseños didácticos $(14,9 \%)$ y los proyectos educativos $(13,4 \%)$. En los porcentajes más bajos se encuentran las tipologías de congresos $(0,5 \%)$, seminarios y planes educativos (1\%) (Gráfico 1). 
Gráfico 1. Porcentajes de las tipologías de los programas educativos

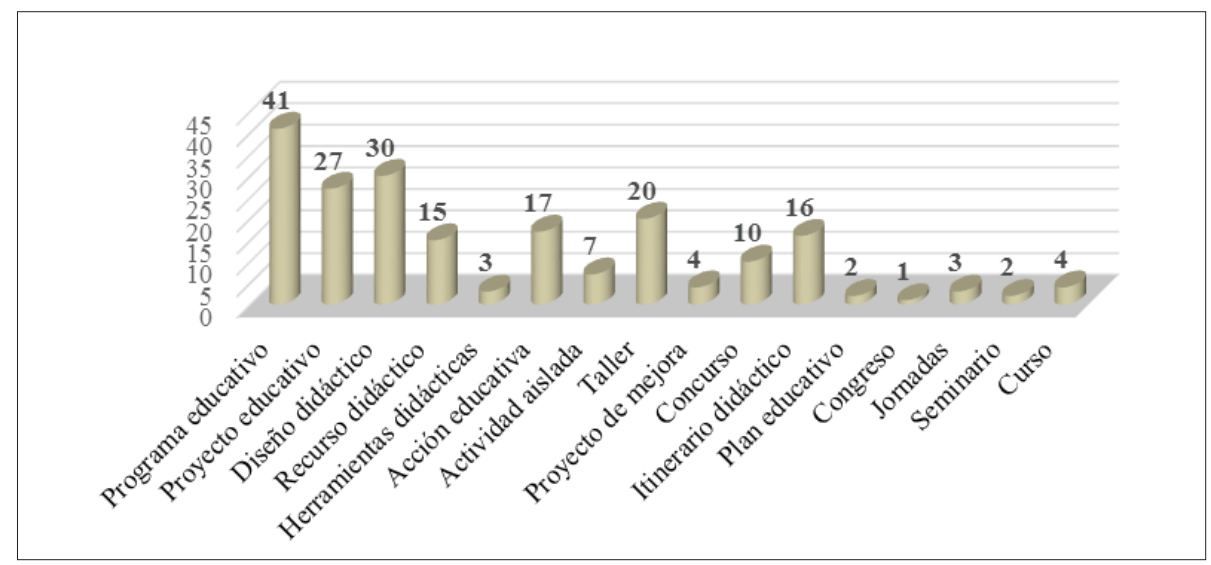

Fuente: elaboración propia.

\section{2. ÁMBITO EDUCATIVO}

Con el fin de acercarnos al ámbito donde se desarrolla la actuación, diferenciamos los siguientes marcos de la actuación (Cuadrado, 2008):

- Educación formal: aprendizajes regulados por instituciones con competencia educativa.

- Educación no formal: acciones educativas no reguladas por normas, sino por el ejercicio de instituciones culturales.

- Educación informal: acciones educativas no pautadas, realizadas por individuos o colectivos.

En la Tabla 3 mostramos los resultados obtenidos en relación con el ámbito educativo dentro del cual se desarrollan los programas.

Tabla 3. Frecuencias y porcentajes de los ámbitos de los programas educativos

\begin{tabular}{|c|l|c|c|c|c|}
\hline \multicolumn{2}{|c|}{ Ámbito de los Programas } & Frecuencia & Porcentaje & $\begin{array}{c}\text { Porcentaje } \\
\text { válido }\end{array}$ & $\begin{array}{c}\text { Porcentaje } \\
\text { acumulado }\end{array}$ \\
\cline { 3 - 6 } & Educación formal & 28 & 13,9 & 13,9 & 13,9 \\
\cline { 2 - 6 } Válidos & Educación no formal & 152 & 75,2 & 75,2 & 89,1 \\
\cline { 2 - 6 } & Educación informal & 22 & 10,9 & 10,9 & 100,0 \\
\cline { 2 - 6 } & Total & 202 & 100,0 & 100,0 & \\
\hline
\end{tabular}

Fuente: elaboración propia. 
El ámbito en el cual se desarrollan con más frecuencia los programas educativos sobre el PCI es en la educación no formal, con una amplia diferencia sobre la educación formal y la educación informal. Así tenemos la educación no formal, con 152 programas (75,2\%); la educación formal, con 28 programas $(13,9 \%)$ y la educación informal con 22 programas, que supone solamente un $10,9 \%$ (Gráfico 2).

Gráfico 2. Porcentajes de las tipologías de los programas educativos

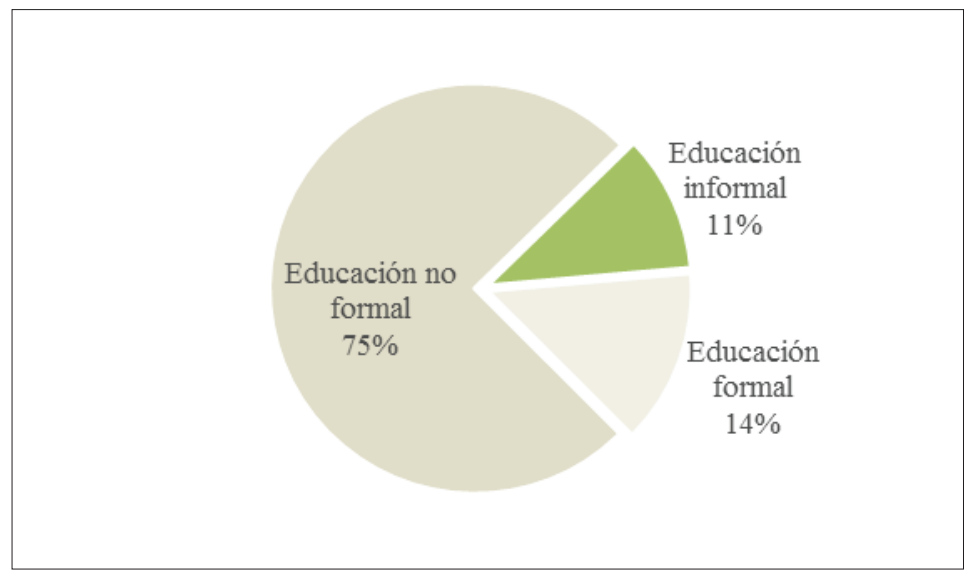

Fuente: elaboración propia.

\subsection{DESTINATARIOS}

Atendiendo a cuál es el público mayoritario al que se dirigen los programas, analizamos los programas teniendo en cuenta dos tipos de público: Alumnado y Público general.

Tabla 4. Frecuencias y porcentajes de tipo de público de los programas educativos

\begin{tabular}{|c|l|c|c|c|c|}
\hline \multicolumn{2}{|c|}{ Tipo de público } & Frecuencia & Porcentaje & $\begin{array}{c}\text { Porcentaje } \\
\text { válido }\end{array}$ & $\begin{array}{c}\text { Porcentaje } \\
\text { acumulado }\end{array}$ \\
\cline { 3 - 6 } & Alumnado & 97 & 48,0 & 48,0 & 48,0 \\
\cline { 2 - 6 } Válidos & Público general & 105 & 52,0 & 52,0 & 100,0 \\
\cline { 2 - 6 } & Total & 202 & 100,0 & 100,0 & \\
\hline
\end{tabular}

Fuente: elaboración propia. 
Con el código Público general, encontramos más de la mitad de los programas educativos, un 52\%, al no especificar el tipo de público al que se dirige se podría inferir que se refiere a programas que abarcan a la mayoría de los destinatarios posibles, puesto que muestra una generalización. El 48\% restante está dirigido hacia el Alumnado. Dentro del alumnado, la etapa educativa con mayor número corresponde a la educación primaria (Gráfico 3).

Gráfico 3. Porcentajes de tipos de público de los programas educativos

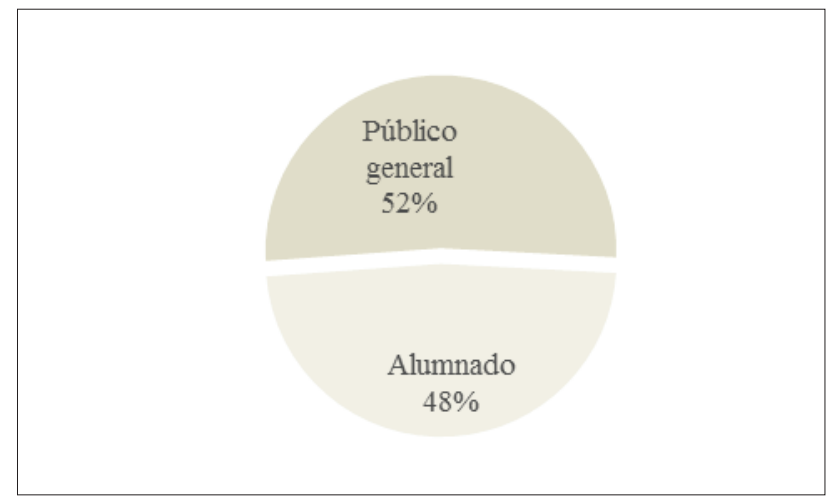

Fuente: elaboración propia.

\subsection{ADAPTACIÓN A PERSONAS CON DIVERSIDAD FUNCIONAL}

En cuanto a los programas dirigidos a personas con diversidad funcional o necesidades educativas especiales, consideramos que el propio concepto de patrimonio sitúa a este como herramienta fundamental para trabajar la diversidad. De acuerdo con Fontal y Marín (2016) es necesario un cambio de paradigma hacia la cultura de la inclusión que entienda la diversidad como una realidad presente en todas las esferas de la sociedad. Por ello, en la Tabla 5 analizamos la frecuencia de programas que presentan adaptación a personas con diversidad funcional. Es decir, Adaptado a personas con diversidad funcional, No adaptado.

Tabla 5. Frecuencias y porcentajes de programas adaptados a personas con diversidad funcional

\begin{tabular}{|l|l|c|c|c|c|}
\cline { 2 - 5 } \multicolumn{2}{c|}{} & Frecuencia & Porcentaje & $\begin{array}{c}\text { Porcentaje } \\
\text { válido }\end{array}$ & $\begin{array}{c}\text { Porcentaje } \\
\text { acumulado }\end{array}$ \\
\hline Válidos & $\begin{array}{l}\text { Adaptado a personas con } \\
\text { diversidad funcional }\end{array}$ & 9 & 4,5 & 4,5 & 4,5 \\
\cline { 2 - 6 } & No habilitado & 193 & 95,5 & 95,5 & 100,0 \\
\cline { 2 - 6 } & Total & 202 & 100,0 & 100,0 & \\
\hline
\end{tabular}


De los 202 programas educativos inventariados solamente 9 están adaptados a personas con diversidad funcional, lo que supone el $(4,5 \%)$ sobre el total. Debemos destacar que, de los 9 programas adaptados a discapacitados, solamente uno de ellos cuenta con recursos humanos (Gráfico 4).

Gráfico 4. Porcentajes adaptación a discapacitados de los programas educativos

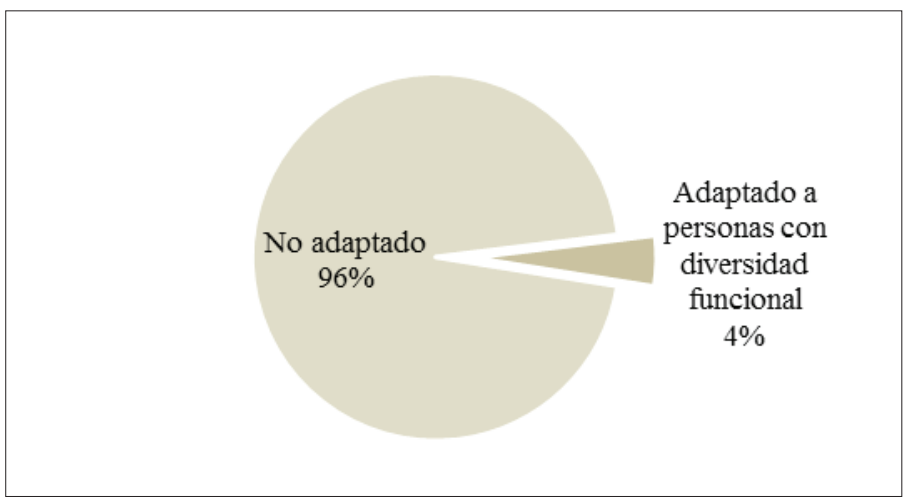

Fuente: elaboración propia.

\subsection{DISEÑO EDUCATIVO}

Para el análisis descriptivo de este apartado relativo a las características del diseño educativo, y con el fin de valorar la estructura didáctica de los programas, hemos clasificado los programas en dos grupos: "con diseño educativo" y "sin diseño educativo" (Tabla 6). Incluimos dentro de los programas con diseño educativo aquellos que plantean y desarrollan los siguientes parámetros:

- Justificación de los planteamientos (si la hay).

- Los objetivos didácticos, atendiendo a la terminología verbal tal como conocer, sensibilizar, valorar comprender y reflexionar.

- Los contenidos patrimoniales.

- La orientación metodológica, activa, participativa, lúdica, práctica, etc.

- $\quad$ Las estrategias de enseñanza aprendizaje.

- Adaptación.

- Temporalización del programa.

- Evaluación del programa y de los resultados del programa con respecto a la adquisición de conocimientos y valores planteados en los objetivos. 
Tabla 6. Frecuencias y porcentajes del diseño educativo de los programas educativos

\begin{tabular}{|c|l|c|c|c|c|}
\hline \multicolumn{2}{|c|}{ Diseño educativo } & Frecuencia & Porcentaje & $\begin{array}{c}\text { Porcentaje } \\
\text { válido }\end{array}$ & $\begin{array}{c}\text { Porcentaje } \\
\text { acumulado }\end{array}$ \\
\cline { 3 - 6 } & Diseño educativo & 117 & 57,9 & 57,9 & 57,9 \\
\cline { 2 - 6 } Válidos & Sin diseño educativo & 85 & 42,1 & 42,1 & 100,0 \\
\cline { 2 - 6 } & Total & 202 & 100,0 & 100,0 & \\
\hline
\end{tabular}

Fuente: elaboración propia.

En lo relativo a los planteamientos metodológicos en los que se fundamentan los programas educativos, únicamente el 57,9\% de los programas presentan diseño didáctico motivo de estudio. Frente al $42,1 \%$ restante que no define un diseño didáctico (Gráfico 5), es decir, que no desarrollan las partes necesarias que conforman un diseño educativo completo, o solo se abordan alguna de sus partes, de manera muy superficial.

Gráfico 5. Porcentajes de diseño educativo de los programas educativos

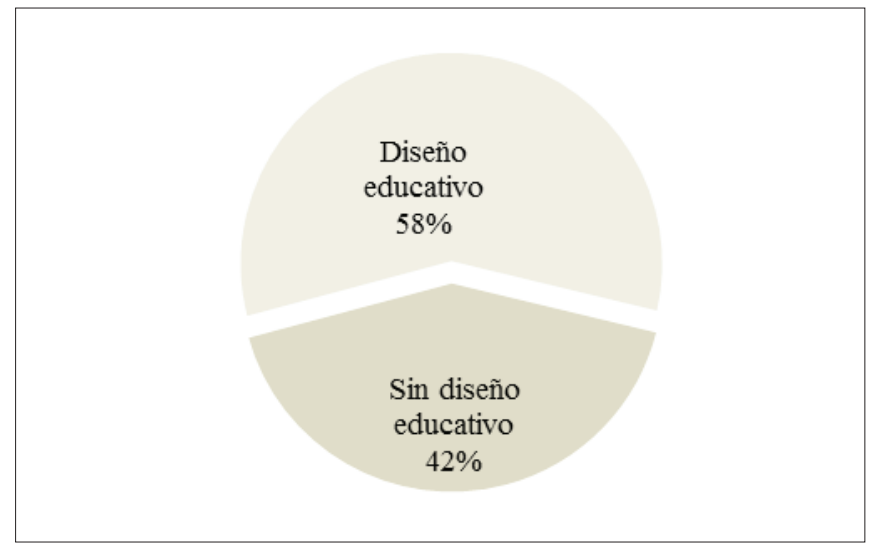

Fuente: elaboración propia.

\subsection{CONTINGENCIAS ENTRE LAS VARIABLES}

A continuación, empleamos la tabla de contingencias para explorar y analizar la relación entre las siguientes variables:

- $\quad$ Tipo de Proyecto * Destinatarios.

- $\quad$ Ámbito educativo * Diseño Educativo. 
En primer lugar, relacionamos la categoría tipo de programa con el tipo de público (Tabla 7).

Tabla 7. Tabla de contingencia Tipo de Proyecto * Tipo de Público

\begin{tabular}{|c|c|c|c|c|}
\hline \multirow{2}{*}{\multicolumn{2}{|c|}{ Recuento }} & \multicolumn{2}{|c|}{ Tipo de público } & \multirow{3}{*}{$\begin{array}{c}\text { Total } \\
41\end{array}$} \\
\hline & & \multirow{2}{*}{$\begin{array}{c}\text { Alumnado } \\
25\end{array}$} & \multirow{2}{*}{$\frac{\text { Público general }}{16}$} & \\
\hline \multirow{16}{*}{ Tipo de Proyecto } & Programa educativo & & & \\
\hline & Proyecto educativo & 10 & 17 & 27 \\
\hline & Diseño didáctico & 28 & 2 & 30 \\
\hline & Recurso didáctico & 6 & 9 & 15 \\
\hline & Herramientas didácticas & 0 & 3 & 3 \\
\hline & Acción educativa & 8 & 9 & 17 \\
\hline & Actividad aislada & 0 & 7 & 7 \\
\hline & Taller & 12 & 8 & 20 \\
\hline & Proyecto de mejora & 0 & 4 & 4 \\
\hline & Concurso & 5 & 5 & 10 \\
\hline & Itinerario didáctico & 2 & 14 & 16 \\
\hline & Plan educativo & 0 & 2 & 2 \\
\hline & Congreso & 0 & 1 & 1 \\
\hline & Jornadas & 0 & 3 & 3 \\
\hline & Seminario & 0 & 2 & 2 \\
\hline & Curso & 1 & 3 & 4 \\
\hline \multicolumn{2}{|l|}{ Total } & 97 & 105 & 202 \\
\hline
\end{tabular}

Fuente: elaboración propia.

En la Tabla 7 podemos apreciar las tipologías de programas cuyos diseños educativos presentan mayor consistencia y conceptualización. Es decir, aquellas tipologías de programas que presentan un diseño educativo planificado y completo se dirigen en mayor medida al alumnado, entre ellos los programas educativos y los diseños didácticos. Por otra parte, los programas de menor conceptualización, o cuya definición no contempla diseño educativo, se desarrollan entre el público general, como son itinerarios didácticos, talleres y jornadas. Debemos destacar la falta de desarrollo de ciertos programas para el alumnado, si bien, dentro de los programas dirigidos al público en general, también podemos encontrar al alumnado. De igual modo, en la representación gráfica (Gráfico 6) podemos observar que ambas líneas de tendencia se muestran ascendentes hacia los programas cuyos diseños educativos son más consistentes; sin embargo, la del alumnado lo hace en mayor medida. 
Gráfico 6. Contingencia Tipo de Proyecto * Tipo de Público

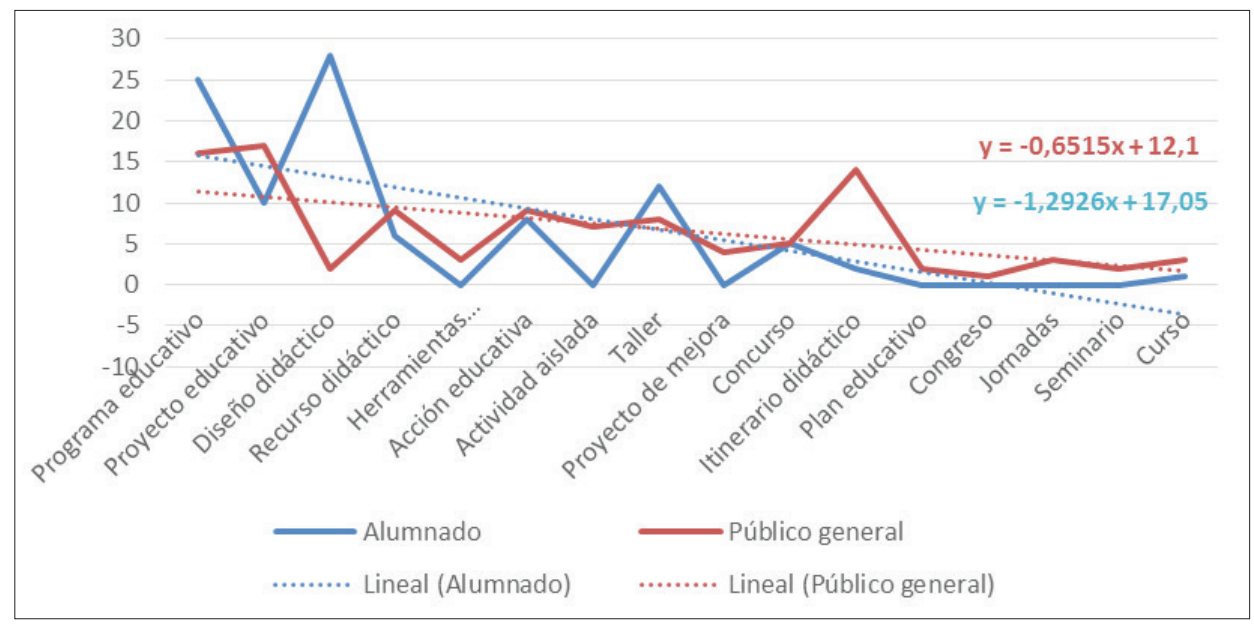

Fuente: elaboración propia.

Mostramos a continuación la relación entre el ámbito educativo, donde son implementados los programas y el diseño educativo (Tabla 8).

Tabla 8. Tabla de contingencia Ámbito de los Programas * Diseño Educativo

\begin{tabular}{|l|l|c|c|c|}
\hline \multirow{2}{*}{ Recuento } & \multicolumn{2}{|c|}{ Diseño educativo } & \multirow{2}{*}{ Total } \\
\cline { 3 - 5 } & $\begin{array}{c}\text { Diseño } \\
\text { educativo }\end{array}$ & $\begin{array}{c}\text { Sin diseño } \\
\text { educativo }\end{array}$ & \\
\hline \multirow{3}{*}{ Ámbito educativo } & Educación formal & 19 & 1 & 20 \\
\cline { 2 - 5 } & Educación no formal & 92 & 65 & 157 \\
\cline { 2 - 5 } & Educación informal & 6 & 19 & 25 \\
\hline Total & 117 & 85 & 202 \\
\hline
\end{tabular}

Fuente: elaboración propia.

En la educación formal, casi la totalidad de los programas presentan diseño educativo. En la educación no formal, existe cierta proporción entre programas con diseño educativo y sin diseño educativo. La educación informal presenta menos programas con diseño educativo. Gráficamente, podemos observar cómo la línea de tendencia diseño educativo, es ascendente hacia la educación formal. En el caso contrario, la línea de tendencia sin diseño educativo es ascendente hacia la educación informal (Gráfico 7). 
Gráfico 7. Contingencia Ámbito educativo de los Programas * Diseño Educativo

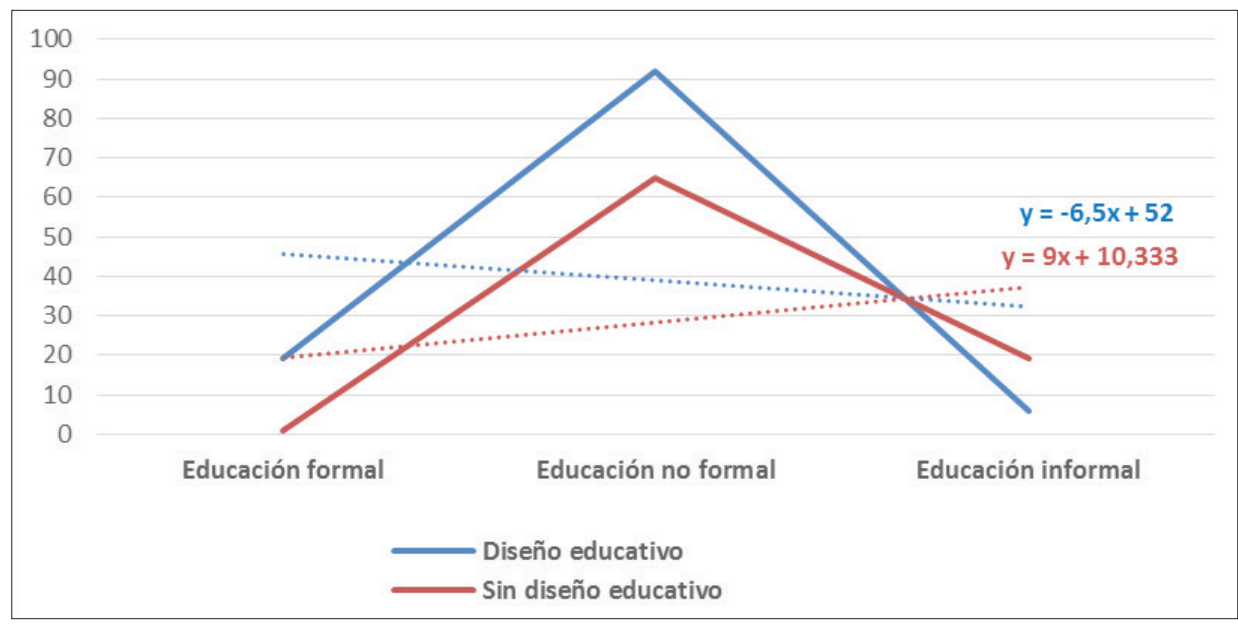

Fuente: elaboración propia.

\section{CONCLUSIONES Y DISCUSIÓN: AUSENCIAS Y PROSPECCIÓN EN LA EDUCACIÓN PATRIMONIAL}

El dinamismo intrínseco en el concepto de patrimonio cultural influye en la evolución del término y deriva en nuevos bienes patrimoniales, como son los bienes inmateriales. Este aspecto también provoca una evolución en el ámbito de la educación patrimonial, con la aparición de programas educativos sobre patrimonio cultural inmaterial que deben ser analizados y evaluados. En este sentido, este artículo ofrece un análisis relacional de los enfoques y categorías de los programas educativos desarrollados en torno al patrimonio inmaterial, que se desarrolla dentro de un proceso evaluativo secuenciado de los mismos, a su vez enmarcado dentro del Observatorio de Educación Patrimonial en España (Fontal, 2016). La investigación ha mostrado que la gran deficiencia detectada es el gran porcentaje de programas analizados que no presentan un diseño educativo estructurado o completo. Es decir, que no desarrollan las partes necesarias que conforman un buen diseño didáctico -fundamentación y adecuación al contexto, objetivos, contenidos, metodología, actividades de enseñanza-aprendizaje, temporalización, adaptación y evaluación-, o son abordadas de manera superficial y muestran limitaciones. Estos resultados coinciden con la investigación llevada a cabo por Marín et al. (2016), donde se presentan limitaciones en el diseño educativo de los programas de educación patrimonial que abordan la inclusión.

Elaborar diseños didácticos sólidos y donde exista concordancia y coherencia entre los contenidos y objetivos planteados en los programas y los contenidos curriculares, es fundamental para dotar a los programas sobre patrimonio de conexiones con los valores, competencias básicas y contenidos que se deben desarrollar en cada etapa como reivindican otros estudios que trabajan en esta área (Cuenca, et al., 2011; Dušan, 2015; Pinto \& Molina, 2015). También debemos destacar de manera negativa la ausencia de programas adaptados a diversos colectivos. Aspecto preocupante si consideramos la diversidad como 
una realidad presente en todas las esferas de la sociedad. Esta postura es compartida en el estudio realizado por Fontal y Martín (2016) que defiende la diversidad presente en el patrimonio y la diversidad de las personas destinatarias de los programas educativos. El uso de las TIC también es escaso en los programas sobre patrimonio inmaterial, a pesar de las ventajas que presenta su utilización, como nos muestran muchas de las investigaciones ya referidas a este respecto (Ibáñez et al., 2012; Ott et al., 2015; Ott \& Pozzi, 2011).

Por otro lado, detectamos ejemplos de buenas prácticas y programas dentro del patrimonio inmaterial que presentan una gran originalidad. Otro aspecto que destacar es la riqueza dentro de la tipología de programa educativo, existiendo representación en todas ellas y mostrando que el patrimonio inmaterial se está trabajando educativamente desde diferentes enfoques. Observamos también bastante representación de programas desarrollados en el ámbito informal y, en menor medida, en el ámbito formal. Además, muchos de ellos se desarrollan en el ámbito formal y en colaboración con otras instituciones de su entorno, aspecto enriquecedor ya que hacen partícipe a todos los agentes educativos, asumiendo lo establecido por el PNEyP como criterio metodológico esencial (Domingo et al., 2013). La participación de los agentes educativos inmersos en el proceso de patrimonialización facilita el proceso de construcción de la identidad, mejorando así la calidad del programa (Fontal \& Gómez-Redondo, 2016). También es fundamental que la legislación educativa aborde de manera sistematizada en todos los niveles la inclusión de contenidos relacionados con el Patrimonio Cultural, su preservación, valoración y disfrute (Fontal \& Martínez, 2016; Fontal et al., 2017), sin olvidar la necesidad de avanzar hacia la coordinación interadministrativa planteada en el PNEyP, que sea capaz de integrar los ámbitos educativos formal, no formal e informal de la educación patrimonial (Cuadrado, 2008).

En cuanto al grado de relación entre categorías que presentan los análisis de contingencias, podemos observar que los programas cuyos diseños educativos son más consistentes presentan un mayor grado de relación con el tipo de público -alumnado-. Por otro lado, aquellos programas con diseño educativo se desarrollan mayoritariamente según las líneas de tendencia en el ámbito de la educación formal, frente a la educación no formal o informal. Estas relaciones pueden deberse a que solamente lo vinculado con el ámbito más formal o escolar se considera que requiere un diseño educativo fundamentado. Esta concepción debe replantearse, ya que toda acción educativa -independientemente del ámbito educativo y del público al cual se dirija- debe estar correctamente planificada y estructurada.

Los programas educativos deben ser diseñados atendiendo en detalle a todos los aspectos que un diseño didáctico -que verdaderamente se haya realizado desde presupuestos propiamente educativos- requiere. Este aspecto es imprescindible para lograr la principal finalidad de los mismos, que es concienciar y estimular el respeto y aprecio al patrimonio (UNESCO 1972, Art. 27 y 28), y activar la cadena de sensibilización (Fontal, 2003) que garantice que el bien cultural, en este caso inmaterial, se convierta en patrimonio para la sociedad, en un referente identitario (Dušan, 2015). Las ausencias y limitaciones detectadas en este análisis deben transformarse en estándares alcanzables, que contengan los diseños de futuros materiales educativos orientados a la transmisión de conceptos y valores patrimoniales sobre el patrimonio inmaterial. Es fundamental que, desde las administraciones, se garantice el desarrollo y consolidación de proyectos de investigación orientados a la consecución de la investigación continua y el seguimiento de acciones desarrolladas en torno a la educación patrimonial (Domingo et al., 2013). Dentro de estos, incluimos el fomento de inventarios y centros de documentación acordes con 
las necesidades de conservación, preservación y difusión orientados a la educación como factor fundamental en la gestión de estos bienes inmateriales. En efecto, la educación patrimonial nos permitirá establecer vínculos identitarios y, desde la experiencia, atribuir significados y valores a los bienes inmateriales transformándolos en patrimonio, para poderlo así conservar y trasmitir (Lobovikov-Katz, 2009).

\section{REFERENCIAS BIBLIOGRÁFICAS}

Angelopoulou, A., Economou, D., Bouki, V., Psarrou, A., Jin, L., Printchar, C., \& Kolyda, F. (2012). Mobile Augmented Reality for Cultural Heritage. Mobile Wireless Middleware, Operating Systems, and Applictions. Springer, 15-22.

Asiáin, A., \& Aznárez, M. (2012). Patrimonio cultural inmaterial y adquisición/desarrollo del lenguaje: Tradición discursiva y psicodinámica oral. Huarte De San Juan, 12, 45-64.

Azmana, N., Halimb, S. A., Liuc, O. P., Saidinb, S., \& Komoo, I. (2010). Public education in heritage conservation for geopark community. Procedia Social and Behavioral Sciences, 7, 504-511.

Barghi, R., Zakaria, Z., Hamzah, A., \& Hashim, N.H. (2017). Heritage education in the Primary School Standard Curriculum of Malaysia. Teaching and Teacher Education, 61, 124-131.

Buckey, J. M., \& Graves D. (2016). Tangible Benefits from Intangible Resources: Using Social and Cultural History to Plan Neighborhood Futures. Journal of The American Planning Association, 82(2), 152-166.

Calaf, R. (2009). Didáctica del patrimonio: epistemología, metodología y estudio de casos. Gijón: Trea.

Carrera, G. (2005). La evolución del patrimonio (inter) cultural: políticas culturales para la diversidad. Colección Cuadernos IAPH, 17, 15-29.

Copeland, T. (2006). European democratic citizenship, heritage education and identity. Strasbourg. France: Council of Europe.

Cuadrado, T. (2008). La enseñanza que no se ve: educación informal en el siglo XXI (Vol. 7). Madrid: Narcea Ediciones.

Cuenca, J. M. (2003). Análisis de concepciones sobre la enseñanza del patrimonio en la educación obligatoria. Revista de Investigación, 2, 37-46.

Cuenca, J. M., Estepa, J., \& Martín, M.J. (2011), El patrimonio cultural en la educación reglada. Revista de Patrimonio Cultural de España, 4, 45-58.

Cuenca, J. M., \& López. I. (2014). Teaching Heritage in Social Science, Geography and History Textbooks in Compulsory Secondary Education. Culture and Education, 26(1), 1-43.

Curtis, R., \& Seymour, C. (2004). Louisiana heritage education program and heritage in the classroom: Children's attitudes toward cultural heritage. Journal of Social Studies Research, 28(2), 20-24.

Deleuze, G., \& Guattari, F. (1972). Lanti-oedipe: capitalisme et schizophrénie. Paris: Editorial De Minuit.

De Troyer, V., Vermeersch, J., VanGenechten, H., Kösten, K., Mols, P., Van Leeuwen, J., . . Vitré, N. (2005). Heritage in the Classroom. A Practical Manual for Teachers (Translated by Julian Morgan). Brussel: Hereduc.

Domingo, M., Fontal, O., \& Ballesteros, P. (2013). Plan Nacional de Educación y Patrimonio. Madrid: MECD, Secretaría de Estado de Cultura.

Dušan, K. (2015). Analysis of National and Cultural Identity Educational Programmes with Examples of Good Practice. Czech-Polish historical and pedagogical journal, 7(2), 72-90.

Estepa, J., Ávila, R. M., \& Ferreras, M. (2008). Primary and Secondary Teachers'Conceptions about Heritage and Heritage Education: A Comparative Analysis. Teaching and Teacher Education, 24, 2095-2107. doi:10.1016/j.tate.2008.02.017 
Falk, H., Dierking, D., \& Adams, M. (2006). Living in a Learning Society: Museums and Free-choice Learning. In S. Macdonald (Ed.), A Companion to Museum Studies (pp. 323-335). Nueva York: Wiley-Blackwell.

Fontal, O. (2016). The Spanish Heritage Education Observatory. Culture and Education, 28(1), 261266.

Fontal, O. (2008). La importancia de la dimensión humana en la didáctica del patrimonio. En S. Mateos (Coord.), La comunicación global del Patrimonio cultural (pp. 79-110). Gijón: Trea.

Fontal, O. (2003). La educación patrimonial. Teoría y práctica en el aula, el museo e internet. Gijón: Trea.

Fontal, O., \& Gómez-Redondo C. (2016). A Quarterly Review of Education. Heritage Education and Heritagization Processes: SHEO Metodology for Educational Programs Evaluation. Interchange, 46(1), 1-26. doi:10.1007/s10780-015-9269-z

Fontal, O., \& Ibáñez, A. (2017). Research on Heritage Education. Evolution and Current State Through analysis of High Impact Indicators. Revista de Educación, 375, 184-214.

Fontal, O., Ibáñez, A., Martínez, M., \& Rivero, P. (2017). El patrimonio como contenido en la etapa de Primaria: del currículum a la formación de maestros. Revista Electrónica Interuniversitaria de Formación del Profesorado, 20(2), 79-94. doi:10.6018/reifop.20.1.286321

Fontal, O., \& Juanola, R. (2015). Heritage Education: a useful and profitable discipline within the cultural heritage management. Revista Cadmo. Giornale Italiano di Pedagogia sperimentale. An International Journal of Educational Research, 23(1), 7-25. doi:10.3280/CAD2015-001002

Fontal, O., \& Marín, S. (2016). Heritage Education in Museums: an Inclusion-Focused Model, International Journal of the Inclusive Museum, 9(4), 47-64.

Fontal, O., \& Martínez, M. (2016). Análisis del tratamiento del Patrimonio Cultural en la legislación educativa vigente, tanto nacional como autonómica, dentro de la educación obligatoria. Madrid: IPCE.

Fontal, O., \& Vallés, J. (2013). Mucho más que... ampliando horizontes para la educación patrimonial. En R. Huerta \& R. de la Calle (Coords.), Patrimonios migrantes (pp. 149-158). Valencia: Servei de publicacions.

Forte, M. (2007). Ecological Cybernetics, Virtual Reality and Virtual Heritage. In F. Cameron \& S. Kenderdine (Eds.), Theorizing digital cultural heritage. A critical discourse (pp. 389-408). Massachussetts: MIT Press.

González, S. (2015). La salvaguarda del patrimonio inmaterial en España (Doctoral Dissertation). Universidad Complutense de Madrid, Madrid.

González, S., \& Querol, M. A. (2014). El patrimonio inmaterial. Madrid: Catarata.

Garner, J., Kaplan, A., \& Pugh, K. (2016). Museums as Contexts for Transformative Experiences and Identity Development. Journal of museum education, 41(4), 341-352.

Haddad, N. (2015). Multimedia and cultural heritage: A discussion for the community involved in children's edutainment and serious games in the 21 st century. Virtual Archaeology Review, 7(14), 61-73.

Hume, D. (1757). La norma del gusto. Valencia: Teoriema, 1980.

Ibáñez, A., Asensio, M., Vicent, N., \& Cuenca, J. M. (2012). Mobile Devices: a Tool for Tourism and Learning at Archaeological Sites. International Journal of Web Based Communities, 8(1), 67-72.

IPCE. (2013). Plan Nacional de Educación y Patrimonio. Madrid: IPCE.

IPCE (2011). Plan Nacional de Salvaguarda del Patrimonio Cultural Inmaterial. Madrid: IPCE.

Jiménez, R.; Cuenca, J. M., \& Ferreras, M. (2010). Heritage education: Exploring the conceptions of teachers and administrators from the perspective of experimental and social science teaching. Teaching and teacher education, 26(6), 1319-1331.

Jin-long, X. (2009). Thinking about Strengthening Undergraduates' Intangible Heritage Education. Journal of Gannan Normal University, 1, 30-54.

Kasapoglu, P. (2016). Applying the Intangible Cultural Heritage Into the Formal Education: A 
WebQuest Example. MILLI FOLKLOR, 111, 149-170.

Lobovikov-Katz, A. (2009). Heritage Education for Heritage Conservation. A Teaching Approach, Strain, 45(1), 480-484.

Lobovikov-Katz, A., Moropoulou, A., Konstanti, A., Ortiz Calderón, P., Van Grieken, R., Worth, S., . . . Cassar, J. (2014). Tangible Versus Intangible in e-learning on Cultural Heritage: From Online Learning to On-site Study of Historic Sites. In Digital Heritage. Progress in Cultural Heritage: Documentation, Preservation, and Protection (pp. 819-828). Switzerland: Springer International Publishing.

Marín, S., García, S., Vicent, N., Gillate, I., \& Gómez-Redondo C. (2017). Inclusive Heritage education in SHEO: a prospective study. Revista de Educación, 375, 110-130. doi:10.4438/1988592X-RE-2016-375-337

Nardi, E. (2008). Education and cultural mediation in museums. Cadmo, 16(2), 1-6.

Ott, M., \& Pozzi, F. (2011). Towards a New Era for Cultural Heritage Education: Discussing the Role of ICT. Computers in Human Behavior, 27(4), 1365-1371.

Ott, M., Dagnino F. M., \& Pozzi, F. (2015). Intangible Cultural Heritage: Towards Collaborative Planning of Educaional Interventions. Computers in Human Behaviour, 51, 1314-1319.

Pinto, H., \& Molina, S. (2015). Heritage Education in the curricula of Social Sciences in Spain and Portugal. Educatio S XXI, 33(1), 103-128.

Rosemary, L., \& Sutter, G. (2012). Sustainability and Museum Education: What Future are we educating for? The International Journal of the inclusive museum, 4(3), 11-26.

Santamarina, C. (2005). Consumo y ocio de los Inmigrantes Latinoamericanos en España: un acercamiento desde la perspectiva cualitativa (Vol. 4). Murcia: Ministerio Trabajo y Asuntos Sociales, Subdirección General de Información Administrativa y Publicaciones.

Soon, C. (2014). ICHPEDIA, a case study in community engagement in the safeguarding of ICH online. International Journal of Intangible Heritage, 9, 69-82.

Stake, E. (2006). Evaluación comprensiva y evaluación basada en estándares (Vol. 10). Barcelona: Graó.

Teixeira, S. (2006). Educación patrimonial: alfabetización cultural para la ciudadanía. Estudios Pedagógicos, 32(2), 133-145.

UNESCO. (1972). Convention Concerning the Protextion of the World Cultural and Natural Heritage. Paris: Author.

UNESCO. (2003). Convention for the Safeguarding of the Intangible Cultural Heritage. 32nd Session of the General Conference, Paris, 29 September-17 October, 2003.

Vicent, N., Rivero, P., \& Feliu, M. (2015). Arqueología y tecnologías digitales en Educación Patrimonial. Educatio Siglo XXI, 33(1), 83-102.

Yanes, C. (2007). El patrimonio educativo intangible: Un recurso emergente en la museología educativa. Cadernos de História da Educaçao, 2, 71-85. 
